\title{
Genomic profiling and predicting treatment response in acute myeloid leukemia
}

\author{
Alessandro Isidori*,1, Federica Loscocco ${ }^{1}$, Antonio Curti ${ }^{2}$, Sergio Amadori ${ }^{3}$ \& Giuseppe \\ Visani ${ }^{1}$ \\ ${ }^{1}$ Hematology \& Hematopoietic Stem Cell Transplant Center, AORMN, Pesaro, Italy \\ ${ }^{2}$ Department of Experimental, Diagnostic \& Specialty Medicine, Institute of Hematology 'L\&A Seràgnoli', University of Bologna, \\ Bologna, Italy \\ ${ }^{3}$ GIMEMA Foundation, 00187 Rome, Italy \\ *Author for correspondence: Tel.: +39 0721 364022; Fax: +39 0721 364052; aisidori@gmail.com
}

“more accurate GEP will probably be part of the diagnostic work-up in the very near future."

First draft submitted: 19 December 2018; Accepted for publication: 5 March 2019; Published online: 24 May 2019

Keywords: acute myeloid leukemia $\bullet$ biomarkers $\bullet$ gene expression profiling $\bullet$ predictive role $\bullet$ prognostication

Acute myeloid leukemia (AML) has a high clinical and biological heterogeneity, characterized by the accumulation of multiple somatically acquired genetic aberrations in hematopoietic stem cells, leading to the impairment of cell growth and differentiation [1].

However, everyday clinical practice is influenced by only a small number of cytogenetic and molecular lesions. In fact, although in the last 15 years the advancement of genomics technologies has produced a wide variety of assays able to identify several molecular alterations underlying the biological heterogeneity of AML, these relevant biological advancements have had weak impact on the development of curative therapies for AML patients. Indeed, even though the identification of molecular mutations within the AML cell population has resulted in better risk stratification, the vast majority of patients are still treated with the same chemotherapy regimens, and allogeneic stem cell transplant still represents the only curative option for intermediate and high-risk AML [2]. Moreover, despite significant progress in leukemia treatment in the last decades, the prognosis of AML still remains poor. The 5-year overall survival (OS) of AML patients, roughly corresponding to the cure rate, since the occurrence of death in CR and late relapses compensate each other, is below $50 \%$ for patients younger than 60 years, and below $20 \%$ for older patients $[2,3]$.

Genetic markers are the best prognosticators in AML, metaphase cytogenetics and somatic mutation assessment by sequencing being included in the current routine workup [4]. It has been known that $55 \%$ of patients have a detectable chromosomal aberration at diagnosis [5,6] since the late 1990s. Now, after the advent of microarray-based gene expression profiling (GEP) and next-generation sequencing (NGS), we know that point mutation and small insertion/deletion, not detected by karyotyping, can be identified in $85 \%$ of patients with normal karyotype by molecular approaches, even those diagnosed with a cytogenetically normal AML $[7,8]$. Gene expression profiling in AML, in fact, has already been demonstrated to be valuable in the diagnosis of different cytogenetic subtypes, discovering novel AML subclasses, and prognostic prediction. Consequently, we have now a better definition of prognosis, which allows us to better stratify patients at diagnosis according to the molecular landscape of AML $[4,9,10]$. Nonetheless, the identification of disease-specific alleles harbored by the malignant clone has, actually, triggered the development of therapies targeting the molecular aberration, such as FLT3 inhibitors, DNMT3A inhibitors, IDH 1 and 2 inhibitors and few others, in order to improve the clinical outcome of AML patients. However, the efficacy of these approaches has proven limited in the long run, due to the clonal evolution of the disease leading to multiple molecular aberrations [11,12]. Accordingly, targeted molecular therapy is still not curative, in the majority of cases, when employed as single therapeutic agent [13].

The major limit of the current approach is that, even if it allows an effective prognostication and patient stratification, it cannot predict response to treatment, referring to single drugs or specific schedules, and cannot give an indication on the selection of patients prone to develop toxicity, basing on pharmacogenomic data [14].

Future $\because$ Medicine 
With this in mind, GEP has recently been profitably used to predict treatment response or resistance to different therapies in several cancers, including AML. Heuser $e t$ al. [15] evaluated the gene expression profiles of hematopoietic stem cells in AML patients receiving chemotherapy. Interestingly, the authors were able to identify a gene-expression signature reflecting molecular differences between responders and nonresponders. The molecular signature predictive of drug resistance was correlated to the gene-expression signature of normal hematopoietic progenitor cells, especially the self-renewing fraction, such as FHL1, MN1 and others [15].

Consistent with this finding, other groups have tried to use GEP assays to predict sensitivity to specific drugs. Tagliafico et al. [16] tested the effect of differentiation therapy with vitamin D or all-trans retinoic acid (ATRA) in both AML cell lines and samples obtained from patients. The aim of their study was to look for the presence of specific gene expression patterns, characterizing the sensitivity of different AMLs to differentiation induction. Interestingly, they identified a gene expression signature potentially able to predict response to ATRA in non-APL AML patients [16]. Unfortunately, the genes identified from this paper were not shown, thereafter, to be good candidates for targeted therapies, but the idea of predicting treatment response by GEP was subsequently utilized by other researchers. The first paper able to predict clinical responses in elderly AML patients treated with targeted drugs was published by Raponi et al. [17] AML patients treated with the farnesyltransferase inhibitor tipifarnib were divided between good and bad responders to tipifarnib basing on the expression of two genes (RASGRP1/APTX). These data were confirmed in an independent group of relapsed/refractory AML patients treated with tipifarnib as single agent [17].

Very recently, our group has shown the prospective use of a GEP-driven therapy in a cohort of hard-to-treat AML patients, unfit for standard therapy, with an extremely poor prognosis [18,19]. 66 unfit AML patients aged $>70$ years received low-dose lenalidomide plus low dose cytarabine $[18,19]$ By studying the global GEP of AML blasts collected before treatment administration, we were able to identify five genes efficiently discriminating patients who did or did not obtain CR. Such signature showed a predictive accuracy of $88 \%$ in the original paper $[18,19]$ Although the 5-gene signature seems to be treatment-specific, and needs a validation in an independent cohort of patients, this is a new scenario for drug development in AML, in which the definition of accurate biomarkers may help discriminate those patients who may or may not benefit from a specific targeted therapy.

An interesting GEP approach to evaluate response to cytarabine in AML patients was recently reported by Lamba et al. [20] Forty-two patients from the AML 97 clinical trial (data set) and 46 patients from the AML 02 clinical trial (validation set), with previously collected microarray GEP data, were studied in order to identify gene-expression signatures able to predict response to cytarabine. With this in mind, the authors performed an integrative and simultaneous analysis of multiple pharmacokinetic, pharmacodynamics (cytarabine dependent) and clinical end points, of potential therapeutic relevance. With a new statistical procedure, called PROMISE [20], candidate probe sets with therapeutically beneficial or detrimental patterns were identified in samples obtained from the data set (AML 97). Thereafter, a PROMISE analysis was made to perform an independent validation of the discovered genes in samples from the validation set (AML 02). Interestingly, the genes identified within the data set, and subsequently validated, play major roles in biological processes relevant to cancer therapy. One of the identified genes was PIK3C3, which exhibits a therapeutically detrimental pattern of association with cytarabine, and is involved in the PI3K/PTEN/Akt/mTOR signaling cascade. This signaling is of major relevance for cell growth and survival. Moreover, the signaling downstream to PI $3 \mathrm{~K}$ is responsible, at least in part, to the development of drug resistance in AML, indicating that the inhibition of PI3K may be useful for a subpopulation of patients with specific expression profiles [20]. Other detrimental genes identified were the HOX genes and AKR1C3 (suppressor of cell differentiation). The unique feature of this study is represented by the identification and validation of genes with a pattern of association with several clinical and pharmacological end points of potential therapeutic relevance.

Finally, another approach to identify patients with an increased risk of developing an adverse reactions, or a lower probability of obtaining a response to cytarabine, is based on the evaluation of genetic variants in key genes involved in its metabolic activation. SNPs and small insertions or deletions in these genes could influence the mRNA expression due to changes in amino acid sequence, and consequently have an influence on the structure of the protein and/or in the activity of the respective gene(s). Thus, SNPs in genes within the metabolic pathway of cytarabine may affect the intracellular level of the drug and its clinical activity, explaining interpatient variability in sensitivity to cytarabine and, accordingly, in the achievement of clinical responses. It is well known that the primary mechanism of cytarabine resistance are the insufficient intracellular levels of the active triphosphate metabolite ara-CTP, that may be due to: low level and/or activity of the transporter (hENT1) involved in the cellular uptake of ara-C; reduced levels of activating enzymes, such as DCK; or increased levels of deactivating enzymes, such as 
NT5C2 and CDA. Functional studies using recombinant DCK proteins have indicated the association of these polymorphisms may negatively affect prognosis by reducing DCK activity, as DCK mRNA expression is positively related to AML treatment outcome. It is necessary, however, to perform in the future validating studies to confirm the possible prognostic relevance of these pharmacogenetics markers.

In conclusion, a more accurate GEP will probably be part of the diagnostic work-up in the very near future. Moreover, studies of GEP may be very helpful to identify gene signatures to be coupled with other prognostic factors, such as cytogenetics/demographics, and used to predict response and adapt therapies to the best match individual patient's leukemia profile. While many validation trials are still ongoing, the potential usefulness of GEP as a sophisticated tool able to possibly identify biomarker(s) is now certainly clear.

\section{Acknowledgements}

The study was supported in part by AlL Pesaro Onlus.

\section{Financial \& competing interests disclosure}

The authors have no relevant affiliations or financial involvement with any organization or entity with a financial interest in or financial conflict with the subject matter or materials discussed in the manuscript. This includes employment, consultancies, honoraria, stock ownership or options, expert testimony, grants or patents received or pending, or royalties.

No writing assistance was utilized in the production of this manuscript.

\section{References}

1. Theilgaard-Monch K, Boultwood J, Ferrari S et al. Gene expression profiling in MDS and AML: potential and future avenues. Leukemia 25, 909-920 (2011).

2. Döhner H, Weisdorf DJ, Bloomfield CD. Acute myeloid leukemia. N. Engl. J. Med. 373(12), 1136-1152 (2015).

3. Isidori A, Venditti A, Maurillo L et al. Alternative novel therapies for the treatment of elderly acute myeloid leukemia patients. Expert. Rev. Haematol. 6(6), 767-784 (2013).

4. Döhner H, Estey E, Grimwade D et al. Diagnosis and management of AML in adults: 2017 ELN recommendations from an international expert panel. Blood. 129(4), 424-447 (2017).

5. Grimwade D, Walker H, Oliver F et al. The importance of diagnostic cytogenetics on outcome in AML: analysis of 1,612 patients entered into the MRC AML 10 trial. The Medical Research Council Adult and Children's Leukaemia Working Parties. Blood 92(7), 2322-2333 (1998).

6. Visani G, Bernasconi P, Boni M et al. The prognostic value of cytogenetics is reinforced by the kind of induction/consolidation therapy in influencing the outcome of acute myeloid leukemia - analysis of 848 patients. Leukemia 15(6), 903-909 (2001).

7. Schlenk RF, Döhner K, Krauter J et al. Mutations and treatment outcome in cytogenetically normal acute myeloid leukemia. N. Engl. J. Med. 358, 1909-1918 (2008).

8. Marcucci G, Mrózek K, Bloomfield CD et al. Molecular heterogeneity and prognostic biomarkers in adults with acute myeloid leukemia and normal cytogenetics. Curr. Opin. Hematol. 12(1), 68-75 (2005).

9. Ley TJ, Miller C, Ding L et al. The Cancer Genome Atlas Research Network. Genomic and epigenomic landscapes of adult de novo acute myeloid leukemia. N. Engl. J. Med. 368(22), 2059-2074 (2013).

10. Papaemmanuil E, Gerstung M, Bullinger L et al. Genomic classification and prognosis in acute myeloid leukemia. N. Engl. J. Med. 374(23), 2209-2221 (2016).

11. Ding L, Ley TJ, Larson DE et al. Clonal evolution in relapsed acute myeloid leukaemia revealed by whole-genome sequencing. Nature 481(7382), 506-510 (2012).

12. Welch JS, Ley TJ, Link DC et al. The origin and evolution of mutations in acute myeloid leukemia. Cell 150(2), 264-278 (2012).

13. Isidori A, Salvestrini V, Ciciarello $\mathrm{M}$ et al. The role of the immunosuppressive microenvironment in acute myeloid leukemia development and treatment. Expert. Rev. Hematol. 7(6), 807-818 (2014).

14. Visani G, Loscocco F, Isidori A, Piccaluga PP. Genetic profiling in acute myeloid leukemia: a path to predicting treatment outcome. Expert. Rev. Hematol. 11(6), 455-461 (2018).

15. Heuser M, Wingen LU, Steinemann D et al. Gene-expression profiles and their association with drug resistance in adult acute myeloid leukemia. Haematologica 90(11), 1484-1492 (2005).

16. Tagliafico E, Tenedini E, Manfredini R et al. Identification of a molecular signature predictive of sensitivity to differentiation induction in acute myeloid leukemia. Leukemia 20(10), 1751-1758 (2006).

17. Raponi M, Lancet JE, Fan $\mathrm{H}$ et al. A 2-gene classifier for predicting response to the farnesyltransferase inhibitor tipifarnib in acute myeloid leukemia. Blood 111(5), 2589-2596 (2008). 
18. Visani G, Ferrara F, Di Raimondo F et al. Low-dose lenalidomide plus cytarabine induce complete remission that can be predicted by genetic profiling in elderly acute myeloid leukemia patients. Leukemia 28(4), 967-970 (2014).

19. Visani G, Ferrara F, Di Raimondo F et al. Low-dose lenalidomide plus cytarabine in very elderly, unfit acute myeloid leukemia patients: final result of a Phase II study. Leuk. Res. 62, 77-83 (2017).

20. Lamba JK, Crews KR, Pounds SB et al. Identification of predictive markers of cytarabine response in AML by integrating analysis of gene-expression profiles with multiple phenotypes. Pharmacogenomics 12(3), 327-339 (2011). 